\title{
Mixed-Integer Programming Model for Ranking Universities: Letting Universities Choose the Weights
}

\author{
Jakub Kudela \\ Institute of Automation and Computer Science, Brno University of Technology, Czech Republic \\ Jakub.Kudela@vutbr.cz
}

\begin{abstract}
Regardless of the shortcomings and criticisms of world university rankings, these metrics are still widely used by students and parents to select universities and by universities to attract talented students and researchers, as well as funding. This paper proposes a new mixed-integer programming model for ranking universities. The new approach alleviates one of the criticisms - the issue of the "arbitrariness" of the weights used for aggregation of the individual criteria (or indicators) utilized in the contemporary rankings. Instead, the proposed model uses intervals of different sizes for the weights and lets the universities themselves "choose" the weights to optimize their position in the rankings. A numerical evaluation of the proposed ranking, based on the indicator values and weights from the Times Higher Education World University Ranking, is presented.
\end{abstract}

Keywords: ranking, university ranking, mixed integer programming, multiplecriteria decision-making.

Received: 10 May 2021 Accepted: 15 June 2021 Published: 21 June 2021

\section{Introduction}

The ranking of universities has in recent years been used as an important tool for universities to publicise their prestige and international positioning [37]. Since the conception of the Academic Ranking of World Universities (ARWU) [23], both the number of university rankings and the number of universities included in them have steadily increased. The growth of these rankings as well as the increase in universities interested in them is explained by the interest of different groups [16].

Universities themselves are eager to occupy dominant positions in these rankings, since this is a way of getting the attention of a greater number of potential students, increasing the revenues from student enrolment [14], and the rankings themselves have an irreducible reputation-making role [26]. The practice of ranking universities has become widely defined by national and international organisations as an important instrument of political and economic policy [3]. This is clearly reflected when ranking positions determine policies related to the restructuring of the higher education system, as in the French case, where different universities were merged to create a university with a high ranking [6]. Despite the shortcomings and the criticism of the university rankings, they are still widely used by students and their parents to select institutions, and by educational institutions to attract talented students and researcher, as well as funding [24]. According to [4], funding explains up to $51 \%$ of the variability of the positions attained by the universities in some rankings.

Apart from the AWRU ranking (or, commonly known as Shanghai ranking), the other prominent rankings are the Times Higher Education (THE) World University Ranking, and the Quacquarelli Symonds (QS) ranking. All three rankings are based on weighing a set of indicators (or dimensions) that should adequately describe the performance of a university in different areas. These indicators typically consist of the quality of education, quality of faculty, number of citations, industry income, international outlook, etc. Recently, new rankings have focused on prominent dimensions not explicitly addressed in the abovementioned rankings, such as innovation (Scimago Institutions Rankings - SIR), web visibility [22], and impact (Webometrics Ranking), or sustainability (GreenMetric World University Ranking). The authors of [10] showed how the intelligent integration of existing data about universities may lead to an open-linked data platform which permits the construction of new indicators, which combine heterogeneous sources of data to generate indicators that address a variety of user requirements without the need to design indicators on a custom basis.

Several researchers have outlined challenging concerns that should be considered before assessing the performance of universities. For instance, some rankings that include survey-based information may bias the results towards those universities that are wellknown compared with lesser-known ones [35]. A factor analysis of the ARWU, QS and THE rankings was performed in [34] and revealed that there were two factors in each of the ranking systems, which did not support the assumption that the indicators were mutually supporting and additive as conceptualised by the ranking providers. The leading global university rankings 
were also explored in [28] to determine the similarities and differences in terms of their ranking criteria, main indicators, modeling choices, and the effects of these on the rankings. In [30], the authors used a sample of Scandinavian universities to show that the differences between the THE and ARWU rankings may be attributed to both small variations on what they believe are not important indicators, as well as substantial variations on what they believe are important indicators. They also provide a methodology that can be used in understanding universities' different ranks in global university rankings. In [7], the authors studied governmentalities of globalizing higher education through a discussion of the competing logics and landscapes of reputation and ranking in two leading universities in South Korea. Their analysis draws attention to the ways in which university rankings have generated a new multi-scalar geography of institutional reputation, the mismatch between quality, reputation and ranking, and the new kinds of institutional behaviors that are emerging to respond to the proliferation of ranking systems. A comparative analysis o five world university rankings was carried out in [27], where it is argued that current rankings are still one-dimensional in the sense that they provide finalized, seemingly unrelated indicator values. The authors of [17] argue that compared to other ranking tools like THE and QS, the ARWU ranking is in many ways "better" - it is simple and transparent, relying on public knowledge and third-party data, not on data provided by the universities themselves or solely on performance data provided by operators like the Institute for Scientific Information. The utilization of rankings and indicators within the universities can also be used as an instrument of new managerialism [25].

Even considering the above shortcomings, there is still a wide consensus that the main weakness of the aforementioned university rankings is linked to the determination of the weights used to measure both dimensions and indicators in the computation of university performance [14]. The combination of multiple indicators of university performance in a single aggregate measure is usually carried out in a rather arbitrary way, which prevents a clear interpretation of the aggregated measure [31, 35]. Also, Using unstandardized indicators for the total scores used in ranking can lead to undesirable results [33]. In the last decade, there have been several approaches proposed to alleviate the aforementioned problems. In [8] the authors proposed a robust ranking of universities, where the aggregation of the university performances is be done by the Choquet integral preference model that is able to take into account the possible negative and positive interactions between the different criteria. A ranking methodology based on ranking hesitant fuzzy sets was developed in [2]. The authors of [29] proposed to measure the importance of a given variable within existing composite indicators via Karl Pearson's "correlation ratio". Hybrid multi-criteria decision making was utilized for ranking
12 private universities in Taiwan in [37]. The composite I-distance indicator methodology as an alternative to weighting was proposed in [12]. In [24], the authors used a contrast pattern mining algorithm to extract a set of patterns describing the top 100 universities in the QS World University Rankings and showed, how are these top universities separable from the rest. A robustness analysis, based on a multi-modelling approach, was performed in [32] to test the validity of the inference about the rankings produced in the ARWU, Shanghai, and THE rankings. The authors conclude that while university and country level statistical inferences are unsound, the inference on macro regions is more robust, and propose an alternative ranking. In [11], the authors proposed a conditional multidimensional approach based on a robust directional distance technique for ranking European universities in teaching and research activities. A goal programming model for the ranking of universities was developed in [14]. Data envelopment analysis model for jointly evaluating the relative teaching and research efficiencies of universities was presented in [18].

In this paper, we propose a new mixed-integer programming model for ranking universities. The model is unique in that it allows each university to "choose" the weights for the aggregation of the individual criteria to get the best possible ranking. It alleviates the issue of of the "arbibtrariness" of the particular values of weights used in different rankings and allows instead for intervals of different sizes for the weights from which the universities can "choose" to optimize their position in the ranking.

The rest of the paper is organised in the following manner. Section 2 introduces the mixed-integer programming model framework for computing the ranking of universities. Section 3 briefly describes the dataset used for the numerical evaluation of the model. Section 4 presents and discusses the empirical results. The main conclusions and implications of the paper are presented in Section 5.

\section{Mathematical Model}

Mixed-integer programming (MIP) is one of the most ubiquitous modelling methods used in optimization [9, 20, 36], with applications ranging from optimal social distancing [19] to optimal plan for the construction of waste processing plants [21].

Our MIP model for ranking universities is based on the following idea: If we let the universities themselves decide on the values of the weights for the individual indicators, how would they choose? Let us consider a situation, where there are $N$ universities to be ranked according to $D$ criteria (or indicators). The values of these criteria are already known and denoted by a vector $p_{i} \in \mathcal{R}^{D}$ for each university $i$. In order to obtain the ranking, we need to determine the weights $w \in \mathcal{R}^{D}$ of the criteria to get a overall score. If we let a particular university $k$ choose these weights, it will naturally set them in such a way that its own ranking is as good 
as possible. This can be achieved by solving the following MIP problem, where $w^{k}$ denotes that the weights are chosen by university $k$ :

$$
\begin{array}{cl}
\text { minimize } & N-\sum_{i=1}^{N} y_{i} \\
\text { subject to } & p_{k}^{\prime} w^{k} \leq p_{i}^{\prime} w^{k}+M y_{i}, \quad \forall i \neq k \\
& p_{k}^{\prime} w^{k} \geq p_{i}^{\prime} w^{k}-M\left(1-y_{i}\right), \quad \forall i \neq k \\
& \sum_{j=1}^{D} w_{j}^{k}=1 \\
& y_{k}=0, \\
& l_{j} \leq w_{j}^{k} \leq u_{j}, \quad \forall j \\
& y_{i} \in\{0,1\}, \quad \forall i
\end{array}
$$

The objective function (1) describes the position of the university $k$ withing the ranking (which should be minimized). The binary variable $y_{i}$ decodes, if the university $i$ is ranked worse than university $k\left(y_{i}=1\right)$ or not $\left(y_{i}=0\right)$. This relationship is enforced by the socalled "Big- $M$ " constraints (2)-(3). The value of the parameter $M$ should be large enough so the conditions hold, but not too large, as it might bring numerical difficulties. Constraint (4) is a normalizing condition on the weights. Constraint (5) forces the ranking to start from position 1 (otherwise, the best ranking university would have position 0). And, finally, constraints (6) and (7) enforce that the weights for the indicators are within pre-specified bounds, and that the variables $y_{i}$ are binary.

After solving the MIP problem (1)-(7) we get the best possible position of the university $k$ (the value of the objective function), and, more importantly, the optimal weights $w^{k}$, which also determine the ranking of all other universities. If we solve MIP problem (1)-(7) for all $N$ universities, we effectively get $N$ observations of possible rankings from which we can easily extract meaningful statistical results. This approach alleviates the issue of the "arbitrariness" of the weights used in the different rankings - instead of a single value, the individual indicators can have a range of values, and the resulting ranking is left "on the universities themselves".

\section{Data}

This section presents the database used to illustrate the implementation of the aforementioned university ranking. Although there is a large variety of rankings currently in use, we chose to apply our model to the data provided by the THE ranking [1]. This ranking is among those with the largest historical data, the number of universities listed in this ranking is very large, and it has the important data readily available at their website.
Table 1 contains a list of the indicators and the weights used in the THE ranking in years 2018-2021. The values of the indicators are standartized: the procedure is based on the distribution of data within a particular indicator, where a cumulative probability function is calculated, using a version of Z-scoring [1]. This means that all values of the indicators fit in a range between 0 (worst performing universities in the indicator) and 100 (best performing universities). The values of the five indicators indicators and their weighted average (Overall) for the top 10 universities in 2021 can be found in Table 2 . The values of the indicators for all considered universities in years 2018-2021 can be found in the supplementary file "DATA.xls".

Table 1: List of indicators used in the THE ranking. [1]

\begin{tabular}{llc} 
Indicator & Definition & Weight \\
\hline \hline \multirow{2}{*}{ International outlook } & $\begin{array}{l}\text { international-to-domestic-student ratio } \\
\text { international-to-domestic-staff ratio } \\
\text { international collaboration }\end{array}$ & $7.5 \%$ \\
\hline Industry income & knowledge transfer & $2.5 \%$ \\
\hline & $\begin{array}{l}\text { reputation survey } \\
\text { staff-to-student ratio } \\
\text { Teaching }\end{array}$ & $\begin{array}{l}\text { doctorate-to-bachelor's ratio } \\
\text { doctorates awarded-to-academic staff ratio }\end{array}$ \\
& institutional income & $30 \%$ \\
\hline \multirow{2}{*}{ Research } & $\begin{array}{l}\text { reputation survey } \\
\text { research income } \\
\text { research productivity }\end{array}$ & $30 \%$ \\
\hline Citations & research influence & $30 \%$ \\
\hline
\end{tabular}

\section{Empirical Results}

We use the indicator values from the THE ranking as a ground for the empirical evaluation of the proposed ranking. The range for the weights is also based on the THE ranking (Table 1 ) and denote them by $w^{T}$. We use a parameter $\alpha$ to denote a possible deviation from the base values, which results in the equation (6) having the following form:

$$
(1-\alpha) w_{j}^{T} \leq w_{j} \leq(1+\alpha) w_{j}^{T}, \quad \forall j .
$$

We compute the results for three different values of $\alpha=[0.3,0.2,0.05]$ and four years: in 2021 there were $N=1527$ universities, in 2020 there were $N=1397$ universities, in 2019 there were $N=1258$ universities, and in 2018 there were $N=1103$ universities considered in the THE ranking. Since the values of the indicators are within 0 and 100, their weighted average will also lie within these abounds. This means that we can set to the value of the "Big $M$ " parameter to $M=100$.

The optimization model was programmed in the high-performance dynamic language JULIA [5] with the JuMP package for mathematical optimization [13]. The solution was computed by the GUROBI 8.0 solver [15]. The computations were carried out on an ordinary computer $(3.2 \mathrm{GHz}$ i5-4460 CPU, 16 GB RAM) and took around five minutes finish for one instance 
Table 2: Indicator and Overall values for the top 10 universities in 2021. [1]

\begin{tabular}{|c|c|c|c|c|c|c|c|}
\hline Rank & Name & Overall & Teaching & Research & Citations & Industry income & International outlook \\
\hline 1 & University of Oxford & 95.6 & 91.3 & 99.6 & 98.0 & 68.7 & 96.4 \\
\hline 2 & Stanford University & 94.9 & 92.2 & 96.7 & 99.9 & 90.1 & 79.5 \\
\hline 3 & Harvard University & 94.8 & 94.4 & 98.8 & 99.4 & 46.8 & 77.7 \\
\hline 4 & California Institute of Technology & 94.5 & 92.5 & 96.9 & 97.0 & 92.7 & 83.6 \\
\hline 5 & Massachusetts Institute of Technology & 94.4 & 90.7 & 94.4 & 99.7 & 90.4 & 90.0 \\
\hline 6 & University of Cambridge & 94.0 & 90.3 & 99.2 & 95.6 & 52.1 & 95.7 \\
\hline 7 & University of California, Berkeley & 92.2 & 85.8 & 97.2 & 99.1 & 84.3 & 72.3 \\
\hline 8 & Yale University & 91.6 & 91.9 & 93.8 & 97.9 & 56.1 & 68.4 \\
\hline 9 & Princeton University & 91.5 & 88.8 & 92.5 & 98.9 & 58.0 & 80.2 \\
\hline 10 & The University of Chicago & 90.3 & 88.9 & 90.5 & 98.6 & 54.9 & 74.0 \\
\hline
\end{tabular}

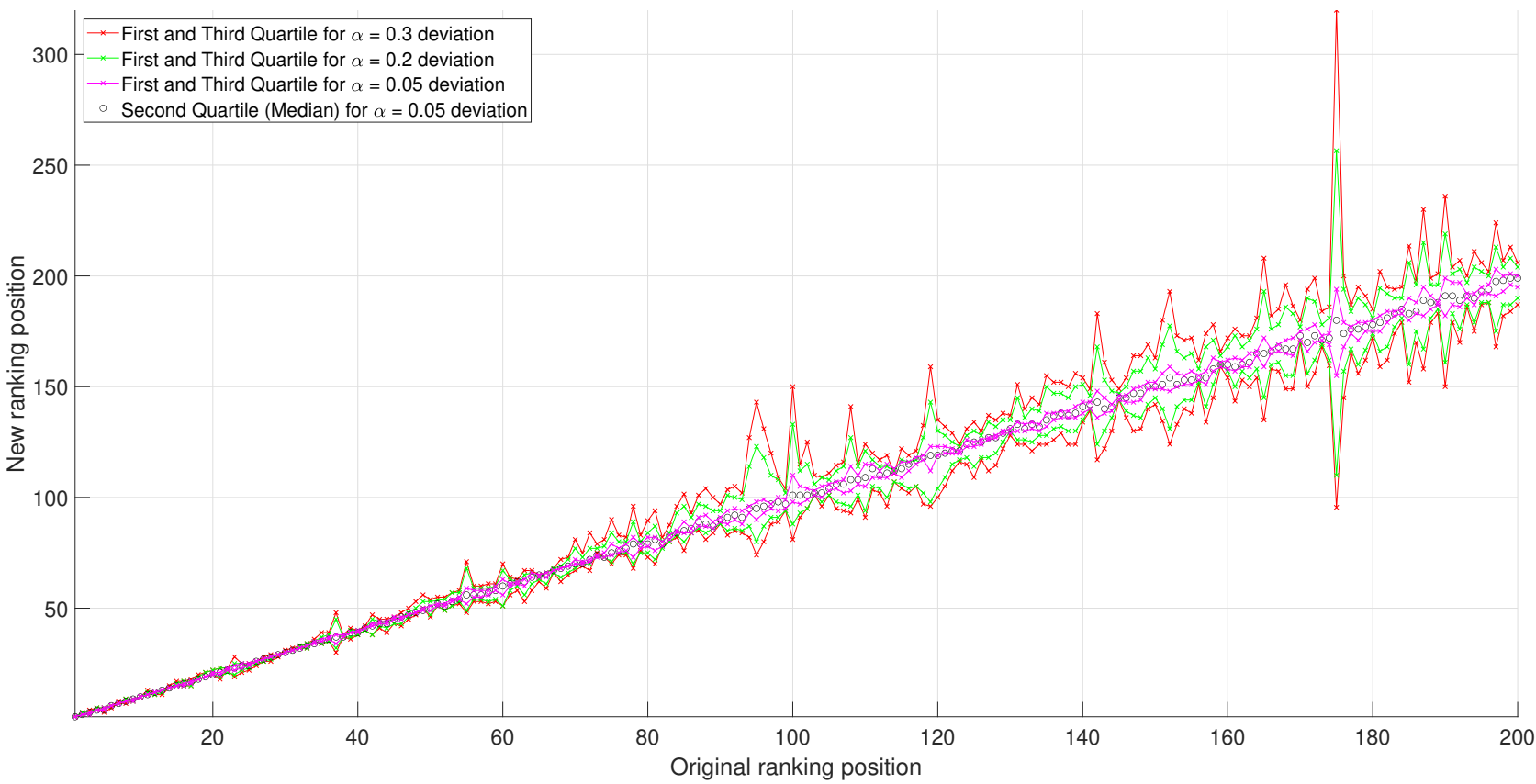

Figure 1: Results of the first 200 universities for different values of $\alpha$, year 2021.

Table 3: Indicator and Overall values for selected universities in 2021. [1]

\begin{tabular}{|c|c|c|c|c|c|c|c|}
\hline Rank & Name & Overall & Teaching & Research & Citations & Industry Income & International Outlook \\
\hline 91 & University of Bristol & 63.0 & 40.3 & 48.6 & 95.6 & 39.8 & 88.2 \\
\hline 92 & University of Basel & 62.9 & 44.0 & 41.4 & 91.7 & 99.7 & 97.2 \\
\hline 92 & University of Glasgow & 62.9 & 40.9 & 48.1 & 94.3 & 39.9 & 92.8 \\
\hline 94 & Purdue University West Lafayette & 62.5 & 57.1 & 65.5 & 62.0 & 69.9 & 71.5 \\
\hline 94 & Zhejiang University & 62.5 & 65.9 & 65.6 & 52.3 & 100.0 & 65.1 \\
\hline 96 & Korea Advanced Institute of Science and Technology & 62.4 & 64.4 & 68.1 & 57.9 & 100.0 & 36.6 \\
\hline 97 & National Taiwan University & 62.3 & 57.1 & 66.7 & 66.9 & 69.5 & 44.8 \\
\hline 170 & University of Leicester & 56.0 & 30.9 & 33.6 & 96.2 & 37.6 & 90.7 \\
\hline 170 & University of Notre Dame & 56.0 & 52.1 & 45.5 & 71.5 & 38.1 & 57.3 \\
\hline 170 & Sant'Anna School of Advanced Studies - Pisa & 56.0 & 45.8 & 39.6 & 79.9 & 85.9 & 57.2 \\
\hline 174 & University of Exeter & 55.9 & 32.4 & 38.3 & 89.9 & 35.8 & 91.5 \\
\hline 174 & Lomonosov Moscow State University & 55.9 & 80.0 & 67.6 & 12.9 & 97.7 & 70.7 \\
\hline 176 & Northeastern University & 55.8 & 36.7 & 29.1 & 98.2 & 36.5 & 76.0 \\
\hline 176 & Ulsan National Institute of Science and Technology & 55.8 & 34.9 & 40.7 & 90.8 & 85.3 & 49.1 \\
\hline 178 & University of Aberdeen & 55.7 & 29.5 & 34.2 & 94.4 & 45.1 & 95.6 \\
\hline 178 & Newcastle University & 55.7 & 31.4 & 38.2 & 90.6 & 40.1 & 88.0 \\
\hline 178 & Paris-Saclay University & 55.7 & 37.3 & 48.7 & 80.4 & 34.5 & 65.1 \\
\hline
\end{tabular}


(solving (1)-(7) for one range on the weights, one year, $N$ times for all universities).

As the relevant statistical information about the resulting ranking of a given university, we chose the first quartile, the second quartile (the median), and the third quartile of its position (out of the $N$ possible rankings that were "chosen" by the $N$ universities). The detailed results of the computations can be found in the supplementary file "RESULTS.xls". In Fig. 1 are shown the statistical information about the first 200 universities for the year 2021 and different values of $\alpha$. We can see that, roughly speaking, the high ranking universities retain their high ranking and have a relatively small difference between the first and the third quartile of their position, even for the largest considered deviation. As the position of the university goes down, this interval between the quartiles widens. An interesting jump in the size of the interval can be seen around universities with original THE ranking position between 91 and 97 . The values of the indicators for these universities are reported in Table 3. The thing the universities with large intervals have in common is a relatively lower value of the "Citations" indicator when compared with other similarly ranked universities (which is compensated by their relatively higher values in "Teaching" and "Research"). Similar, but a bit more dramatic effect can be seen between the positions 170 and 180, where the "Lomonosov Moscow State University" stands out with a large difference between the first and third quartile, which is caused by its poor value of the "Citations" indicator.

Although the size of the interval is higher for lower ranking universities, it does not grow to unreasonable values. The sizes of the intervals for different values of $\alpha$ and different years can be seen in Fig. 2. Naturally, the higher the allowed deviation $\alpha$, the larger the size of the intervals. The THE ranking reports a "precise" rank for the first 200 universities and groups the rest into ranges of 201-250, 251-300, 301-350, 351-400, 401-500, 501-600, 601-800, 801-1000, and 1000+ (i.e., 1001-1527 for the year 2021). The ranking proposed obtained by the proposed method results in a smaller ranges, as can be seen in Table 4 . The effect of the proposed ranking on the top 100 universities in years 2018-2021 can be seen in Table 5 .

\section{Conclusion}

Despite their problems and criticisms, rankings of the world universities are here to stay, and will go on to be used by students, parents, researchers, and funding agencies, for categorizing and selecting universities. In this paper, we proposed a mixed-integer programming model for ranking universities that aims to alleviate one of the criticisms faced by contemporary university rankings - the "arbitrariness" of the weights used for aggregate scores. We have shown, that by using intervals for the weights instead and letting the universities themselves choose the particular values of the weights from these intervals to optimize their position in the rankings, we can get a reasonable ranking. We have demonstrated the properties of the model on a numerical example that was based on the THE ranking in years the 2018-2021. The top-performing universities still occupy the best positions regardless of the approach followed by the mixed-integer programming model, confirming their leadership. For the other universities, the mixed-integer model provides a meaningful range on their ranking based on the difference between the third and first quartile of their position in all possible rankings (chosen by all the universities). These ranges are comparatively smaller than the ones reported in the THE ranking, even for large values of the allowed deviation $\alpha$. Although the "arbitrariness" of the weights is alleviated, it is not completely removed, as the intervals for the possible values of the weights still have to be agreed upon.

Acknowledgement: This work was supported by IGA Brno University of Technology project No. FSIS-20-6538.

\section{References}

[1] Times higher education university ranking, 2021. https://www.timeshighereducation.com/worlduniversity-rankings/ [Accessed 26th Apr 2021].

[2] Alcantud, J., de Andrés Calle, R., And Torrecillas, M. Hesitant fuzzy worth: An innovative ranking methodology for hesitant fuzzy subsets. Applied Soft Computing 38 (2016), 232243.

[3] Amsler, S., And Bolsmann, C. University ranking as social exclusion. British Journal of Sociology of Education 33 (2012), 283-301.

[4] Benito, M., Gil, P., And Romera, R. Funding, is it key for standing out in the university rankings? Scientometrics 121 (2019), 771-792.

[5] Bezanson, J., Edelman, A., Karpinski, S., AND SHAH, V. Julia: a fresh approach to numerical computing. SIAM Review 59 (2017), 65-98.

[6] Billaut, J., Bouyssou, D., And Vincke, P. Should you believe in the shanghai ranking? Scientometrics 84 (2009), 237-263.

[7] Collins, F., And Park, G. Ranking and the multiplication of reputation: reflections from the frontier of globalizing higher education. Higher Education 72 (2016), 115-129.

[8] Corrente, S., Greco, S., And SŁowiński, R. Robust ranking of universities evaluated by hierarchical and interacting criteria. In Huber S., Geiger M., de Almeida A. (eds) Multiple Criteria Decision Making and Aiding. International Series in Operations Research \& Management Science (2019), pp. 145-192.

[9] DA Silva, R. F., Hvattum, L. M., AND Glover, F. Combining solutions of the optimum satisfiability problem using evolutionary tunneling. MENDEL Journal 26 (2020), 23-29. 

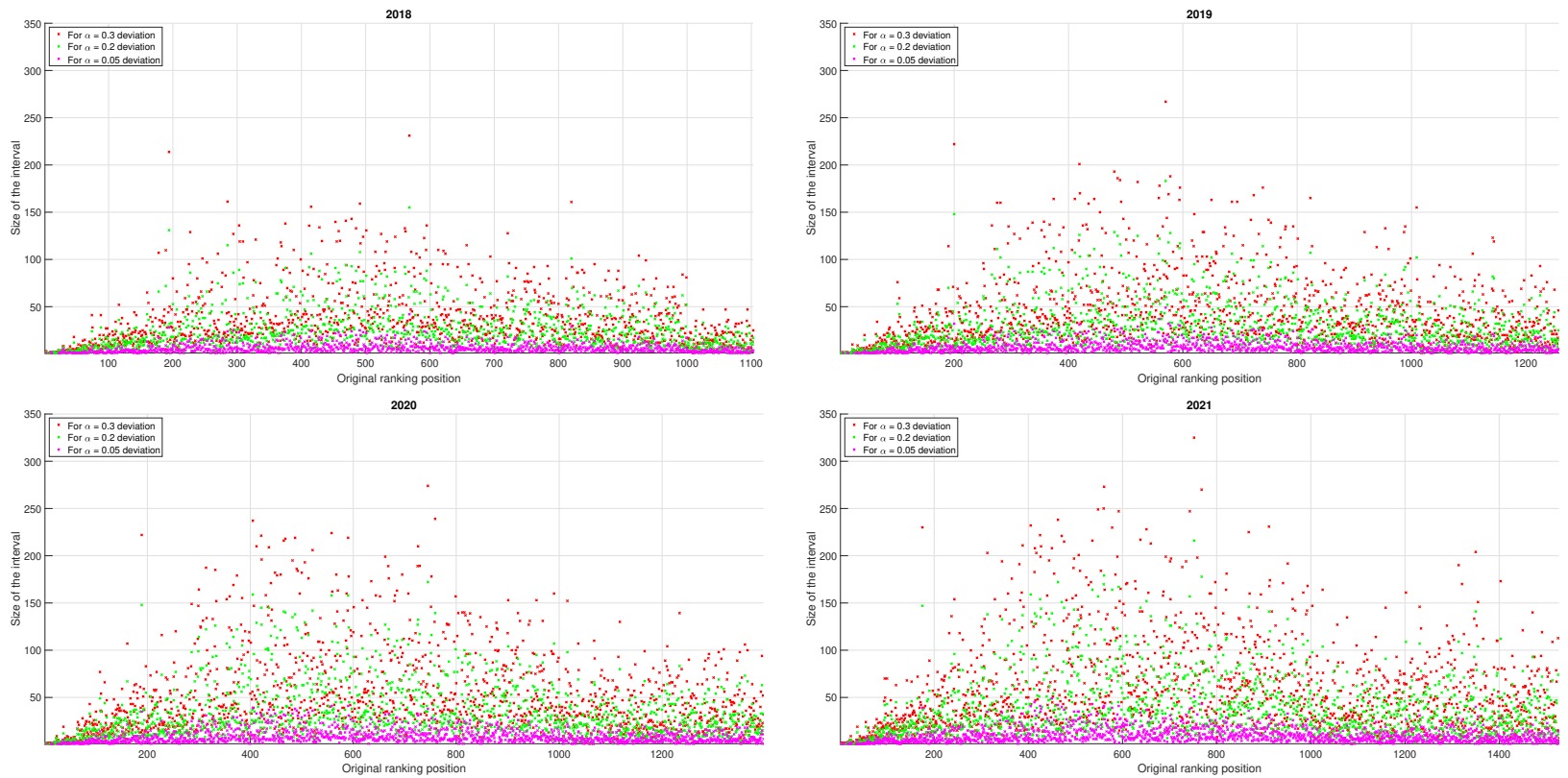

Figure 2: Size of the interval between the first and the third quartile for different values of $\alpha$ and different years.

Table 4: Interval sizes (difference between the first and third quartile) for different values of $\alpha$ and different years.

\begin{tabular}{l|rrr|rrr} 
& \multicolumn{4}{|c|}{ median interval size } & \multicolumn{4}{|c}{ maximum interval size } \\
& $\alpha=0.3$ & $\alpha=0.2$ & $\alpha=0.05$ & $\alpha=0.3$ & $\alpha=0.2$ & $\alpha=0.05$ \\
\hline \hline 2018 & 28 & 19 & 5 & 231 & 155 & 42 \\
2019 & 34 & 22 & 6 & 267 & 183 & 49 \\
2020 & 39 & 25 & 6 & 274 & 172 & 41 \\
2021 & 44 & 29 & 7 & 325 & 216 & 51
\end{tabular}

[10] Daraio, C., And Bonaccorsi, A. Beyond university rankings? generating new indicators on universities by linking data in open platforms. Journal of the Association for Information Science and Technology 68 (2017), 508-529.

[11] Daraio, C., Bonaccorsi, A., And Simar, L. Rankings and university performance: A conditional multidimensional approach. European Journal of Operational Research 244 (2015), 918-930.

[12] Dobrota, M., Bulajic, M., Bornmann, L., AND JeREMIC, V. A new approach to the qs university ranking using the composite i-distance indicator: Uncertainty and sensitivity analyses. Journal of the Association for Information Science and Technology 67 (2016), 200-211.

[13] Dunning, I., Huchette, J., And Lubin, M. Jump: a modeling language for mathematical optimization. SIAM Review 59 (2017), 295-302.

[14] García, F., Guijarro, F., And Oliver, J. A multicriteria goal programming model for ranking universities. Mathematics 9 (2021), 459.

[15] GUROBI. Gurobi optimizer 8.0, 2021. http://www.gurobi.com/ [Accessed 26th Apr 2021].

[16] Johnes, J. University rankings: What do they really show? Scientometrics 115 (2018), 585-606.

[17] KAUPPI, N. The global ranking game: narrowing academic excellence through numerical objectifi- cation. Studies in Higher Education 43 (2018), 1750-1762.

[18] KuAh, C., AND Wong, K. Efficiency assessment of universities through data envelopment analysis. Procedia Computer Science 3 (2011), 499-506.

[19] Kudela, J. Social distancing as p-dispersion problem. IEEE Access 8 (2020), 149402-149411.

[20] Kudela, J., And Popela, P. Two-stage stochastic facility location problem: Ga with benders decomposition. MENDEL 21 (2015), 53-58.

[21] Kudela, J., Smejkalova, V., Somplak, R., AND NEVRLY, V. Legislation-induced planning of waste processing infrastructure: A case study of the czech republic. Renewable and Sustainable Energy Reviews 132 (2020), 110058.

[22] Lages, J., Patt, A., And Shepelyansky, D. Wikipedia ranking of world universities. The European Physical Journal B 89 (2016), 69.

[23] Liu, N., And Cheng, Y. The academic ranking of world universities. Higher Education in Europe 30 (2005), 127-136.

[24] Loyola-GonzÁlez, O., Medina-PÉrez, M., VAlDEZ, R., AND CHOO, K.-K. A contrast pattern-based scientometric study of the qs world university ranking. IEEE Access 8 (2020), 206088-206104. 
Table 5: The values of the first, the second (in bold), and the third quartile for the top 100 universities in years 2018-2021, allowed deviation $\alpha=0.3$.

\begin{tabular}{|c|c|c|c|c|c|c|c|c|c|}
\hline & 2021 & 2020 & 2019 & 2018 & & 2021 & 2020 & 2019 & 2018 \\
\hline University of Oxford & $1: 1: 1$ & $1: 1: 1$ & $1: 1: 1$ & $1: 1: 1$ & UoM & $51: 51: 54$ & $56: 55: 58$ & $55: 56: 58$ & $53: 54: 57$ \\
\hline Stanford University & $2: 2: 3$ & $2: 4: 4$ & $2: 3: 3$ & $3: 3: 3$ & University of Sydney & 49:51:55 & $56: 60: 61$ & $58: 59: 64$ & 60:61:65 \\
\hline Harvard University & $2: 3: 4$ & $5: 7: 7$ & $5: 6: 6$ & 6:6:6 & UoSC & $52: 53: 57$ & $62: 62: 65$ & $62: 66: 68$ & $60: 66: 68$ \\
\hline CalTech & $4: 4: 5$ & $2: 2: 3$ & $3: 5: 5$ & $2: 3: 5$ & Boston University & 52:54:58 & 59:61:64 & $68: 74: 83$ & 64:70:77 \\
\hline MIT & $3: 5: 5$ & $5: 5: 5$ & $4: 4: 5$ & $4: 5: 5$ & Kyoto University & $47: 54: 71$ & $52: 65: 76$ & $54: 65: 88$ & 58:74:99 \\
\hline University of Cambridge & $5: 6: 6$ & $2: 3: 4$ & $2: 2: 3$ & $2: 2: 4$ & CUoHK & $53: 56: 60$ & $54: 57: 59$ & 51:53:55 & $58: 58: 61$ \\
\hline $\mathrm{UoCB}$ & $7: 7: 8$ & $13: \mathbf{1 3}: 14$ & $14: 15: 15$ & $17: 18: 19$ & THKUoSaT & $54: 56: 60$ & $44: 47: 49$ & 40:41:46 & $41: 44: 46$ \\
\hline Yale University & $7: 8: 9$ & $8: 8: 8$ & $8: 8: 8$ & 10:12:12 & UoNCaCH & $52: 56: 61$ & 54:54:59 & $55: 57: 59$ & $53: \mathbf{5 6}: 62$ \\
\hline Princeton University & 8:9:9 & 6:6:6 & $7: 7: 7$ & $7: 7: 7$ & ANL & 54:59:61 & $48: 50: 53$ & $47: 49: 52$ & $46: 48: 50$ \\
\hline The University of Chicago & 10:10:10 & 9:9:10 & 9:10:10 & 8:9:9 & SNU & 51:60:69 & $54: 64: 71$ & $56: 63: 77$ & 63:74:91 \\
\hline Imperial College London & 11:11:13 & 9:10:11 & 9:9:10 & $8: 8: 9$ & Brown Univer & $57: 61: 64$ & $53: 53: 58$ & $51: 53: 54$ & $47: 50: 52$ \\
\hline Johns Hopkins University & 11:12:12 & $11: \mathbf{1 2}: 12$ & 11:12:12 & $13: 13: 14$ & TUoQ & $59: 62: 63$ & $65: 66: 68$ & $67: 69: 74$ & 63:65:66 \\
\hline University of Pennsylvania & $11: \mathbf{1 3}: 13$ & 10:11:12 & $11: \mathbf{1 3}: 13$ & 10:10:11 & WU\&R & $54: 62: 67$ & $55: 59: 63$ & $57: 59: 66$ & $59: 64: 68$ \\
\hline ETH Zurich & 14:14:15 & 13:13:14 & 11:11:13 & 10:10:12 & UoCD & 58:64:67 & $55: 55: 59$ & 61:59:62 & $53: 54: 55$ \\
\hline UoCLA & $15: \mathbf{1 5}: 17$ & $15: \mathbf{1 7}: 17$ & $17: \mathbf{1 7}: 17$ & $14: \mathbf{1 5}: 16$ & Monash University & 62:64:65 & $75: 75: 82$ & $82: 84: 89$ & $80: 80: 82$ \\
\hline University College Lor & $15: 16: 17$ & $15: 15: 17$ & 14:14:15 & $15: 16: 17$ & University of Amsterdam & $60: 66: 66$ & $61: 62: 65$ & $59: 62: 65$ & $56: 59: 64$ \\
\hline Columbia University & $15: 17: 18$ & $15: \mathbf{1 6}: 17$ & $15: \mathbf{1 6}: 16$ & $13: 14: 15$ & UNSW Sydney & $66: 67: 68$ & 69:71:74 & 90:96:99 & $81: 85: 90$ \\
\hline$y$ of Toronto & 18:18:20 & $18: \mathbf{1 8}: 19$ & 20:21:22 & $22: \mathbf{2 2}: 23$ & UoCSB & $62: 68: 72$ & $53: 57: 61$ & $47: \mathbf{5 2}: 56$ & $49: \mathbf{5 3}: 59$ \\
\hline Cornell University & 19:19:21 & 19:19:19 & 18:19:19 & 18:19:19 & McMaster University & $65: 69: 73$ & $67: 72: 83$ & $70: 77: 90$ & $76: \mathbf{7 8 : 8 5}$ \\
\hline Duke University & $20: 20: 23$ & $20: \mathbf{2 0}: 23$ & 18:18:19 & $16: 17: 17$ & Fudan University & $66: 70: 80$ & $89: 109: 125$ & 87:104:126 & $94: 116: 146$ \\
\hline Tsinghua University & $18: 20: 23$ & $20: \mathbf{2 3}: 27$ & $18: \mathbf{2 2}: 28$ & $25: 30: 33$ & Leiden University & 69:70:75 & $66: 67: 70$ & $66: 68: 71$ & $67: 67: 72$ \\
\hline UoMAA & $22: \mathbf{2 2}: 23$ & $21: \mathbf{2 1}: 22$ & 20:20:21 & $21: \mathbf{2 1}: 23$ & EUR & $67: 72: 85$ & 64:69:79 & $63: \mathbf{7 0 : 8 0}$ & $66: 72: 78$ \\
\hline Peking University & 19:23:28 & $20: \mathbf{2 4}: 29$ & $25: 31: 34$ & $25: \mathbf{2 7}: 33$ & University of Montreal & $75: 73: 79$ & $82: 85: 93$ & 88:90:95 & 104:108:113 \\
\hline Northwestern University & $21: \mathbf{2 4 : 2 5}$ & $21: \mathbf{2 2}: 24$ & $23: \mathbf{2 5}: 26$ & $20: \mathbf{2 0}: 22$ & University of Zurich & $73: 73: 82$ & $86: 90: 100$ & 87:92:99 & 124:136:145 \\
\hline NUoS & $22: \mathbf{2 5}: 25$ & $24: 25: 28$ & $23: \mathbf{2 3}: 27$ & $20: \mathbf{2 2}: 24$ & CUB & 71:75:91 & $73: 80: 95$ & 83:90:99 & $117: \mathbf{1 2}$ \\
\hline New York University & $24: \mathbf{2 6}: 26$ & $26: \mathbf{2 9}: 28$ & $25: \mathbf{2 7}: 28$ & $27: \mathbf{2 7}: 29$ & Utrecht University & $74: 75: 84$ & $74: 75: 83$ & 69:74:81 & $68: 68: 72$ \\
\hline LSoEaPS & $26: \mathbf{2 7}: 28$ & $25: \mathbf{2 7}: 27$ & $24: \mathbf{2 6}: 27$ & $26: \mathbf{2 6}: 29$ & University of Warwick & $75: 77: 84$ & $74: 77: 85$ & $76: 79: 87$ & 88:90:96 \\
\hline Carnegie Mellon Un & $26: \mathbf{2 8}: 29$ & $24: 27: 29$ & $22: \mathbf{2 4}: 26$ & $22: \mathbf{2 4}: 25$ & DUoT & $68: 78: 95$ & :67:81 & $55: \mathbf{5 8 : 6 4}$ & $58: 63: 73$ \\
\hline University of Washington & $28: \mathbf{2 9}: 29$ & $24: \mathbf{2 6}: 26$ & $26: \mathbf{2 8}: 29$ & $26: \mathbf{2 5}: 28$ & University of Tübingen & $76: 78: 83$ & 90:91:101 & $87: \mathbf{8 9 : 9 2}$ & 92:94:99 \\
\hline University of Edinburgh & $30: 30: 31$ & 29:30:31 & 28:29:30 & $26: \mathbf{2 7}: 30$ & University of Groningen & $74: 80: 91$ & $69: 73: 84$ & $74: 79: 89$ & $78: 82: 87$ \\
\hline University of Melbourne & $31: \mathbf{3 1 : 3 2}$ & $32: \mathbf{3 2 : 3 4}$ & $31: \mathbf{3 2 : 3 3}$ & $31: \mathbf{3 2}: 32$ & HUB & $70: 80: 93$ & $67: 73: 95$ & $63: 67: 76$ & $60: 62: 69$ \\
\hline LMU Munich & $32: \mathbf{3 2}: 33$ & $32: \mathbf{3 2}: 33$ & $32: \mathbf{3 2}: 33$ & $34: \mathbf{3 4 : 3 6}$ & OSUMC & $78: 80: 82$ & $68: 70: 72$ & $71: 71: 74$ & 69:70:73 \\
\hline UoCSD & $32: \mathbf{3 3}: 34$ & $29: 31: 32$ & 29:30:31 & $28: 31: 31$ & University of Freiburg & $80: 83: 88$ & $87: 85: 92$ & $76: \mathbf{7 6 : 8 0}$ & $81: 82: 84$ \\
\hline $\mathrm{UoBC}$ & $35: \mathbf{3 4 : 3 6}$ & $34: 34: 37$ & $36: \mathbf{3 7}: 39$ & $33: \mathbf{3 4 : 3 7}$ & University of Copenhagen & 82:84:96 & 91:101:105 & 110:116:130 & 103:109:124 \\
\hline King's College London & $34: 35: 39$ & $35: \mathbf{3 6}: 39$ & $37: \mathbf{3 8}: 40$ & $35: \mathbf{3 6}: 39$ & Emory University & $78: 85: 102$ & $72: 80: 96$ & $75: 84: 97$ & 84:98:111 \\
\hline Karolinska Institute & $35: \mathbf{3 6}: 40$ & $37: 41: 44$ & $39: 40: 44$ & $36: \mathbf{3 8}: 40$ & University of Minnesota & $84: 85: 93$ & $78: 79: 85$ & 69:71:76 & $55: 56: 60$ \\
\hline The University of Tokyo & $29: \mathbf{3 6}: 48$ & $30: \mathbf{3 6}: 49$ & $35: 43: 55$ & $38: 45: 56$ & École Polytechnique & $85: 87: 102$ & 86:93:109 & 99:108:128 & 107:115:124 \\
\hline GIoT & $37: \mathbf{3 8}: 38$ & $37: \mathbf{3 8}: 39$ & $32: \mathbf{3 4}: 36$ & $31: \mathbf{3 3 : 3 4}$ & UoSaToC & $79: 87: 102$ & $71: 80: 96$ & 79:92:102 & 115:132:142 \\
\hline University of Hong Kong & $36: \mathbf{3 9 : 4 1}$ & $35: \mathbf{3 5}: 40$ & $35: \mathbf{3 6}: 41$ & $34: 40: 42$ & Sorbonne University & $83: \mathbf{8 7 : 1 0 1}$ & $78: 80: 91$ & 69:73:82 & $\mathrm{N} / \mathrm{A}$ \\
\hline McGill University & $38: 40: 40$ & $41: 42: 42$ & $43: 45: 46$ & $42: 42: 44$ & UoMCP & 88:90:97 & 89:91:97 & $81: 83: 88$ & $66: 69: 72$ \\
\hline TUoM & 40:41:42 & $41: 43: 44$ & $43: 45: 45$ & $41: \mathbf{4 1 : 4 3}$ & University of Bristol & 84:91:104 & $77: 87: 101$ & 70:77:90 & 69:76:79 \\
\hline Heidelberg University & $38: 42: 47$ & $40: \mathbf{4 4}: 49$ & $43: 47: 50$ & $43: 45: 48$ & University of Basel & $86: 92: 105$ & $86: 94: 107$ & 98:103:109 & 90:95:106 \\
\hline EPFdL & $41: 43: 45$ & $37: \mathbf{3 8}: 39$ & $34: 35: 37$ & $36: \mathbf{3 8}: 41$ & University of Glasgow & 85:92:103 & $87: 98: 109$ & 82:92:103 & $76: 80: 87$ \\
\hline University of Texas at Austin & $39: 44: 45$ & $35: \mathbf{3 8 : 4 1}$ & $36: \mathbf{3 9 : 4 1}$ & $46: 49: 50$ & PUWL & 81:94:127 & $74: 88: 117$ & $61: 64: 73$ & $53: 60: 67$ \\
\hline KU Leuven & $43: 45: 46$ & $45: 45: 48$ & $48: 48: 50$ & $46: 47: 49$ & Zhejiang University & 74:94:144 & 74:107:151 & 67:101:143 & $127: \mathbf{1 7 7}: 234$ \\
\hline Paris Sciences et Lettres & $41: \mathbf{4 6 : 4 8}$ & $42: 45: 50$ & $39: 42: 45$ & $70: 72: 75$ & KAIST & 79:96:129 & 83:110:137 & 83:102:117 & $84: 95: 111$ \\
\hline NTUS & $45: 47: 51$ & $45: 49: 49$ & $48: \mathbf{5 1 : 5 3}$ & $49: 52: 55$ & NTU & $87: 97: 120$ & 99:120:146 & 138:171:206 & 166:198:246 \\
\hline UoIaUC & $47: 48: 53$ & $44: 48: 49$ & $48: 50: 52$ & $36: \mathbf{3 7}: 39$ & UoCI & 89:98:110 & $83: 96: 109$ & $83: 96: 102$ & 90:99:109 \\
\hline UoWM & $50: 49: 54$ & $47: 51: 51$ & $41: 44: 46$ & $41: 43: 45$ & University of Helsinki & 93:98:104 & 91:96:102 & 93:99:100 & 89:90:94 \\
\hline WUiSL & $46: 50: 54$ & $47: \mathbf{5 2 : 5 4}$ & $51: 55: 57$ & $47: 50: 53$ & SJTU & 79:100:150 & $111: \mathbf{1 5 7}: 218$ & $133: \mathbf{1 8 8}: 247$ & 142:188:252 \\
\hline
\end{tabular}

California Institute of Technology (CalTech), Massachusetts Institute of Technology (MIT), University of California - Berkeley (UoCB), University of California - Los Angeles (UoCLA), University of Michigan-Ann Arbor (UoMAA), National University of Singapore (NUoS), London School of Economics and Political Science (LSoEaPS), University of California - San Diego (UoCSD), University of British Columbia (UoBC), Georgia Institute of Technology (GIoT), Technical University of Munich (TUoM), École Polytechnique Fédérale de Lausanne (EPFdL), Nanyang Technological University - Singapore (NTUS), University of Illinois at Urbana-Champaign (UoIaUC), University of Wisconsin-Madison (UoWM), Washington University in St Louis (WUiSL), University of Manchester (UoM), University of Southern California (UoSC), Chinese University of Hong Kong (CUoHK), The Hong Kong University of Science and Technology (THKUoSaT), University of North Carolina at Chapel Hill (UoNCaCH), Australian National University (ANL), Seoul National University (SNU), The University of Queensland (TUoQ), Wageningen University \& Research (WU\&R), University of California - Davis (UoCD), University of California - Santa Barbara (UoCSB), Erasmus University Rotterdam (EUR), Charité - Universitätsmedizin Berlin (CUB), Delft University of Technology (DUoT), Humboldt University of Berlin (HUB), Ohio State University - Main campus (OSUMC), University of Science and Technology of China (UoSaToC), University of Maryland - College Park (UoMCP), Purdue University West Lafayette (PUWL), Korea Advanced Institute of Science and Technology (KAIST), National Taiwan University (NTU), University of California Irvine (UoCI), Shanghai Jiao Tong University (SJTU) 
[25] LYNCH, K. Control by numbers: new managerialism and ranking in higher education. Critical Studies in Education 56 (2015), 190-207.

[26] Marginson, S. University rankings and social science. European Journal of Education 49 (2014), 45-59.

[27] Moed, H. A critical comparative analysis of five world university rankings. Scientometrics 110 (2017), 967-990.

[28] Olcay, G., and Bulu, M. Is measuring the knowledge creation of universities possible?: A review of university rankings. Technological Forecasting and Social Change 123 (2017), 153-160.

[29] Paruolo, P., Saisana, M., and Saltelli, A. Ratings and rankings: voodoo or science? Journal of the Royal Statistical Society Series A (Statistics in Society) 176 (2013), 609-634.

[30] Piro, F., and Sivertsen, G. How can differences in international university rankings be explained? Scientometrics 109 (2016), 2263-2278.

[31] Rahnamayan, S., Mahdavi, S., Deb, K., and BIDGOLI, A. Ranking multi-metric scientific achievements using a concept of pareto optimality. Mathematics 8 (2020), 956.

[32] Saisana, M., D'Hombres, B., and Saltelli, A. Rickety numbers: Volatility of university rankings and policy implications. Research Policy 40 (2011), 165-177.

[33] SoH, K. Misleading university rankings: cause and cure for discrepancies between nominal and attained weights. Journal of Higher Education Policy and Management 35 (2013), 206-214.

[34] SoH, K. What the overall doesn't tell about world university rankings: examples from arwu, qswur, and thewur in 2013. Journal of Higher Education Policy and Management 37 (2015), 295-307.

[35] Waltman, L., Calero-Medina, C., Kosten, J., Noyons, E., TiJssen, R., van Eck, N., van Leeuwen, T., van RaAn, A., Visser, M., AND Wouters, P. The leiden ranking 2011/2012: Data collection, indicators, and interpretation. Journal of the American Society for Information Science and Technology 63 (2012), 2419-2432.

[36] Williams, H. Logic and Integer Programming. Springer, Verlag, 2009.

[37] Wu, H.-Y., Chen, J.-K., Chen, I.-S., And Zhuo, H.-H. Ranking universities based on performance evaluation by a hybrid mcdm model. Measurement 45 (2012), 856-880. 